\title{
Bronchopleurobiliary fistula following right lower lobectomy in a patient with prior hepatic abscess: a case report
}

\author{
Jong Hui Suh, Chan Beom Park \\ Department of Thoracic and Cardiovascular Surgery, Incheon St. Mary's Hospital, The Catholic University of Korea, Seoul, Republic of Korea \\ Correspondence to: Chan Beom Park, MD, PhD. Department of Thoracic and Cardiovascular Surgery, Incheon St. Mary's Hospital, College of Medicine, \\ The Catholic University of Korea, 222 Banpo-daero, Seocho-Gu, Seoul, Republic of Korea. Email: drcs5223@daum.net; drcs5223@catholic.ac.kr.
}

\begin{abstract}
In a 68-year-old male patient with cholangitis microabscess in the liver, cavitary lung cancer of the right lower lobe was incidentally diagnosed. The patient's medical history comprised totally laparoscopic distal gastrectomy (TLDG) and cholecystectomy 9 years ago. Before TLDG, endoscopic retrograde cholangiopancreatography (ERCP) was performed because of a common bile duct stone and cholangitis. Three months ago, he was readmitted with cholangitic microabscess, and a cavitary lesion of right lower lobe was detected incidentally. Hepatobiliary microabscess was improved with ERCP and antibiotic treatment. Video-assisted thoracoscopic surgery (VATS) right lower lobectomy and mediastinal lymph node dissection were undergone uneventfully. Pneumonic infiltration in the right lower lung field and hydropneumothorax on the right side were developed on postoperative day 10. Chest computed tomography showed pneumobilia and micro-bronchopleural fistula (BPF) was suspected with bronchoscopic examination. Despite antibiotics usage and thoracic suction, air leakage could not be improved. VATS Talc pleurodesis was performed on postoperative day 38. Contralateral pneumonia developed 1 week following talc pleurodesis. Furthermore, bilirubin was detected in the repeated bronchial washing and pleural fluid and they showed the same color and nature. Though ERCP and endoscopic nasobiliary drainage, the patient's condition deteriorated and the patient passed away on postoperative day 60 . Bronchopleurobiliary fistula is an extremely rare complication after thoracic surgery for which surgical treatment is challenging, early recognition of a developing fistula and the aggressive treatment at an early stage can reduce the associated morbidity and mortality from the sequelae of this rare disease entity.
\end{abstract}

Keywords: Bronchopleurobiliary fistula; complication; lobectomy; case report; hepatic abscess

Submitted Mar 23, 2020. Accepted for publication Sep 17, 2020.

doi: $10.21037 / \mathrm{atm}-20-2776$

View this article at: http://dx.doi.org/10.21037/atm-20-2776

\section{Introduction}

A thoracobiliary fistula is a rare clinical manifestation of biliary disruption, which is caused by infection, trauma, neoplasm, and postoperative complications of hepatobiliary surgery (1-4). They are pathologic communications between the biliary channel and the bronchial tree or the biliary tract and the pleural space.

Hepatopulmonary fistulae follows the erosion of diaphragm due to several causes such as congenital malformations, penetrating liver trauma, hepatobiliary surgery, intrapulmonary rupture of a hepatic hydatid cyst and biliary obstruction with infected suppuration. Other ways of fistula formation involve the expansion of an infected biloma with biliary stasis into the diaphragm. Extensive suppurative process leads to rupture and erosion of diaphragm and invades the pleural space, bronchus or both.

Here we present the rare case of a 68 -year-old male patient with a history of hepatic microabscess who developed a bronchopleurobiliary fistula after right lower lobectomy.

We present the following article in accordance with the CARE reporting checklist (available at http://dx.doi. org/10.21037/atm-20-2776). 

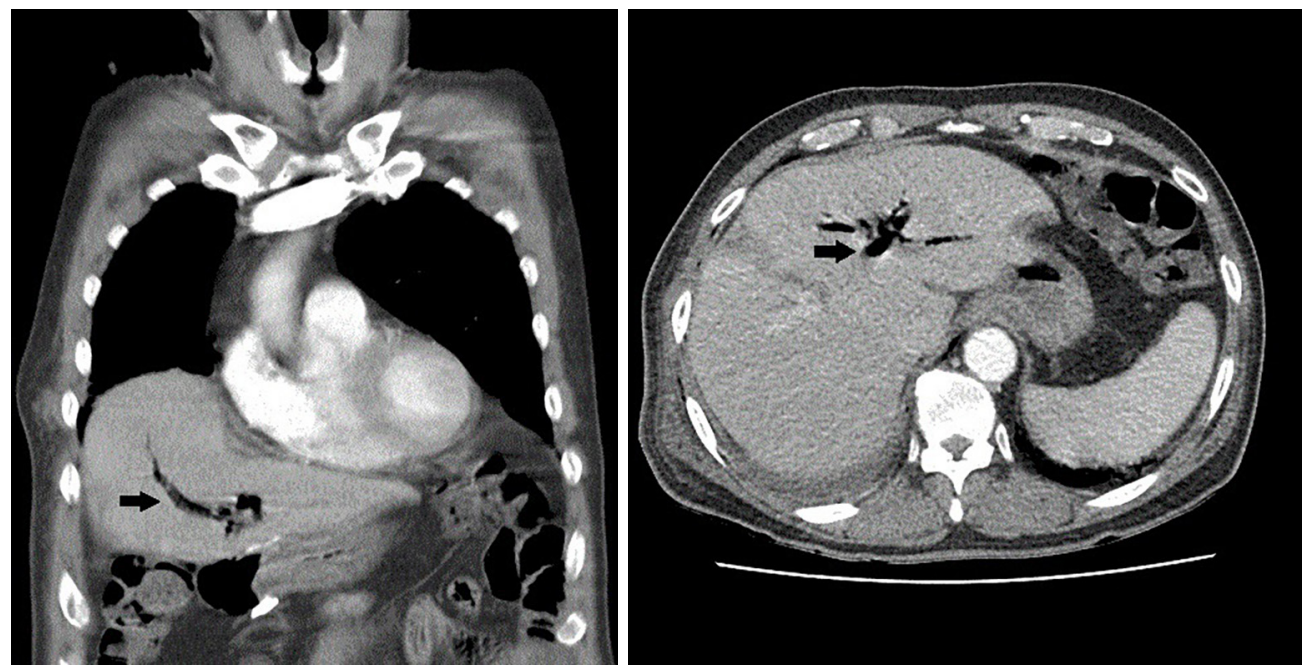

Figure 1 The axial and coronal views of chest computed tomography show recently detected pneumobilia (arrow).

\section{Case presentation}

A 68-year-old male with a history of diabetes and hypertension was referred to our department for the management of cavitary lung lesion of the right lower lobe. His medical history comprised early gastric cancer 9 years ago, which was treated with totally laparoscopic distal gastrectomy (TLDG) and cholecystectomy. Before TLDG, he underwent endoscopic retrograde cholangiopancreatography (ERCP) because of a common bile duct stone and cholangitis. Three months ago, he was readmitted with cholangitic microabscess in the liver S8, and a cavitary lesion of the right lower lobe was detected incidentally. The initiation of ERCP and antibiotic treatment improved the patient's condition, and followup liver dynamic computed tomography (CT) revealed the regression of cholangitic microabscess and pneumobilia was not shown. However, a pulmonary nodule in the right lower lobe was enlarged, from $2.3 \mathrm{~cm}$ at 2 months before chest CT to $2.6 \mathrm{~cm}$.

Upon the current admission, physical examination revealed no rebound tenderness or muscle guarding in the abdomen. On presentation, the patient's blood pressure was $110 / 90 \mathrm{mmHg}$, pulse rate was $80 \mathrm{~min}^{-1}$, respiratory rate was $16 \mathrm{~min}^{-1}$, and body temperature was $36.2^{\circ} \mathrm{C}$. In addition, laboratory findings were as follows: white blood cell count of

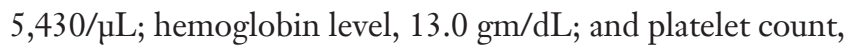
$209,000 / \mu L$. Furthermore, serum AST/ALT levels were normal at $18 / 11 \mathrm{U} / \mathrm{L}$, total bilirubin level was $0.4 \mathrm{mg} / \mathrm{dL}$, C-reactive protein level was $2.12 \mathrm{mg} / \mathrm{L}$, and elevated sedimentation rate was $32 \mathrm{~mm} / \mathrm{h}$.

Accordingly, we performed video-assisted thoracoscopic surgery (VATS) right lower lobectomy and mediastinal lymph node dissection uneventfully. We observed no enlarged lymph node in the mediastinum and no abnormality in the diaphragmatic surface. The postoperative course was uneventful; therefore, the patient was planned to be discharged. On postoperative day 10 , the patient developed fever, pneumonic infiltration in the right lower lung field, and hydropneumothorax on the right side. We performed bronchoscopy and suspected microbronchopleural fistula (BPF). Chest CT revealed recently developed pneumobilia (Figure 1). We administered antibiotics intravenously to the patient, reinserted the chest tube, and applied the $20-\mathrm{mmHg}$ thoracic suction. Despite persistent air leakage, the patient's condition improved gradually. On postoperative day 28, the total bilirubin levels in the serum and chest tube drainage were 0.7 and $0.5 \mathrm{mg} / \mathrm{dL}$, respectively. On postoperative day 33, follow-up bronchoscopic examination revealed BPF aggravation. On postoperative day 38, we performed VATS Talc pleurodesis. In addition, severe pleural adhesion was noted, particularly on the posterior pleural space, and the bronchial stump stapling site was observed, but the air leakage was not prominent and bile was not found. Despite performing Talc pleurodesis, the air leakage persisted, and the thoracic suction was continued. After 1 week of VATS pleurodesis, the patient developed contralateral pneumonia. Meanwhile, the patient developed yellowish sputum and repeated bronchoscopic examination on postoperative 


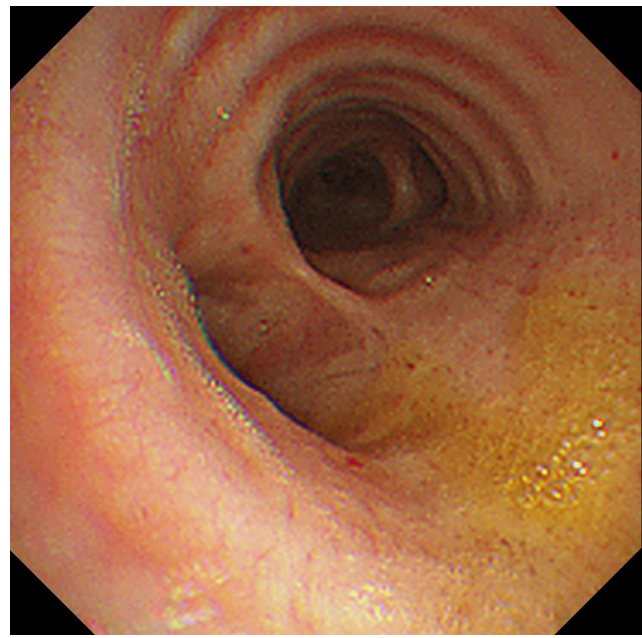

Figure 2 Bronchoscopic examination shows a yellowish material coating the right lower bronchus.

day 47 confirmed the bile-stained sputum (Figure 2). The total bilirubin levels in the serum, chest tube drainage, and bronchial washing were $0.8,0.8$, and $0.3 \mathrm{mg} / \mathrm{dL}$, respectively and bronchial washing and pleural fluid showed the same color and nature (Figure 3).

Methicillin-resistent Staphylococcus aureus and vancomycin-resistent enterococcus isolated from the pleural fluid and bronchial washing culture was positive for imipenem-resistent Acinetobacter baumannii.

However, the patient's condition deteriorated abruptly, resulting in his transfer to the intensive care unit. On day 48, we performed ERCP and endoscopic nasobiliary drainage (ENBD) because of a bronchopleurobiliary fistula. However, the patient's condition aggravated despite ERCP and $\mathrm{ENBO}$, and he died on postoperative day 60.

All procedures performed in studies involving human participants were in accordance with the ethical standards of the institutional and/or national research committee(s) and with the Helsinki Declaration (as revised in 2013). Written informed consent was obtained from the patient families.

\section{Comments}

Thoracobiliary fistulas are rare pathologic communications between the biliary tract and the bronchial tree (bronchobiliary fistulas) or the biliary tract and the pleural space (pleurobiliary fistulas). Previously, several studies have reported bronchobiliary and pleurobiliary fistulas

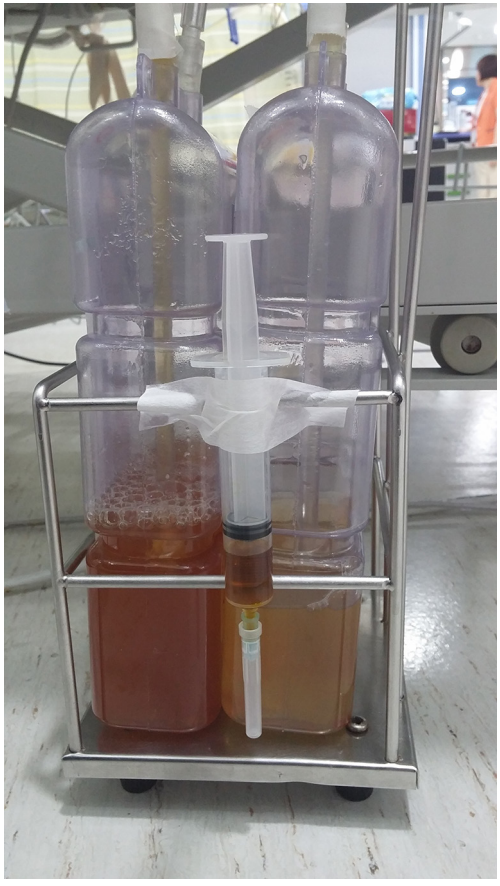

Figure 3 Bronchial washing and pleural fluid showing the same color and nature.

(1-4); however, as per our knowledge, the occurrence of a bronchopleurobiliary fistula after pulmonary resection has not been reported to date.

The occurrence of acquired bronchobiliary fistula could be attributed to several causes, including hepatic hydatid disease or liver abscess (echinococcic, amebic, or pyogenic), blunt or penetrating traumatic biliary tract obstruction, iatrogenic fistulas after liver resection, radiofrequency ablation, bile duct stricture, irradiation, and thoracic drainage. Both primary and secondary hepatic tumors are frequent preexisting clinical conditions (5), and chronic pancreatitis, subphrenic abscess, and acute cholecystitis in a suprahepatic gall bladder can cause a bronchobiliary fistula. Typically, bronchobiliary or pleurobiliary fistulas are known to originate from the hepatobiliary system; however, in the present study, these characteristically originated from a $\mathrm{BPF}$ after right lower lobectomy in a patient with potential hepatic microabscess.

Although biliptysis and pneumonia were the leading clinical manifestations and jaundice was a rare finding, it could be misdiagnosed as acute pneumonitis or a chronic irritable cough producing greenish sputum, as in our patient. Predictably, ERCP or percutaneous transhepatic cholangiography facilitated direct visualization of the 
fistula. However, contrast-enhanced magnetic resonance cholangiography and hepatobiliary iminodiacetic acid (HIDA) scan are more advantageous than conventional noninvasive techniques.

To date, two mechanisms of pathogenesis have been suggested (4). First, the biliary tract obstruction results in the retention of bile and a liver biloma eventually leads to the abscess formation. Usually, an increased abscess erodes the diaphragm, lung parenchyma, and bronchus and causes a bronchobiliary fistula. Second, a thoracobilary fistula occurs without the biliary tract obstruction. Amebic, or pyogenic liver abscess enlarges and erodes the diaphragm, which results in the formation of a bronchobiliary or thoracobilary fistula. Unlike these mechanisms, the fistula in our patient originated from the BPF after right lower lobectomy. The BPF eroded the diaphragm, and the continuous thoracic suction with negative pressure augmented an irritation on the pleural space and diaphragmatic surface. In our patient, the BPF could have been a potential infection source of hepatic microabscess. In addition, diaphragmatic pores could be communicating sources between the pleural and abdominal cavities. The negative thoracic suction and negative inspiratory pressure during respiration exacerbated the bile regurgitation into the bronchial tree through the pleural space and caused a bronchopleurobiliary fistula.

Apparently, bile exerts a corrosive effect on the lungs and pleural space. While a pleurobiliary fistula predisposes a loculated bilious empyema, a bronchobiliary fistula may predispose to a necrotizing bronchitis, bronchopneumonia, or chronic indirect pneumonitis. Therefore, early diagnosis with a high index of clinical suspicion is imperative for the optimal management. Both bronchoscopic examination and the illustration of the bile-stained secretion in the bronchial tree are crucial for the diagnosis. In addition, a biliary excretion scintigraphic (HIDA) scan can visualize the contrast material in the biliary tree and direct communication with the bronchial tree. Prodromos et al. suggested that elevated bilirubin levels in the sputum and/ or pleural effusion (range, $4-10 \mathrm{mg} / \mathrm{dL}$ ) were diagnostic criteria $(2,6)$. However, in our patient, the total bilirubin levels in pleural effusion, serum, and bronchial washing were $0.8,0.8$, and $0.3 \mathrm{mg} / \mathrm{dL}$, respectively, signifying that the low bilirubin level in pleural effusion and bronchial washing could not exclude the diagnosis of bronchopleurobiliary fistula.

To date, the optimal treatment remains controversial and challenging. Previously, less invasive techniques, such as endoscopic retrograde biliary drainage, endoscopic sphincterotomy, ENBD, endoscopic stone extraction, and percutaneous transhepatic cholangial drainage, have been performed. The surgical treatment of pulmonary lobectomy, closure of fistula tract, hepatic lobectomy, hepaticoenterostomy, and abscess drainage alone can also be considered. In the case of diaphragmatic, pleural, bronchial, or pulmonary damages, closure of the diaphragm, pleural drainage, decortication, or different pulmonary resections can be performed (7-9). Although recurrent bronchobiliary fistula has rarely been reported after surgical management, it is typically reoperative and related to substantial morbidity and mortality.

The limitation of the present study is that communications between bronchus, pleural space and biliary trees are not confirmed with imaging studies such as HIDA scan. Bronchopleurobiliary fistula can be hardly suspected at early stage, therefore, early suspicion and definitive diagnostic procedure are important for patient outcome.

In conclusion, bronchopleurobiliary fistula is a sporadic complication after thoracic surgery for which surgical treatment is challenging, and early clinical suspicion is imperative for the management of bronchopleurobiliary fistula.

\section{Acknowledgments}

Funding: None.

\section{Footnote}

Reporting Checklist: The authors have completed the CARE reporting checklist (available at http://dx.doi.org/10.21037/ atm-20-2776).

Conflicts of Interest: All authors have completed the ICMJE uniform disclosure form (available at http://dx.doi. org/10.21037/atm-20-2776). The authors have no conflicts of interest to declare.

Ethical Statement: The authors are accountable for all aspects of the work in ensuring that questions related to the accuracy or integrity of any part of the work are appropriately investigated and resolved. All procedures performed in studies involving human participants were in accordance with the ethical standards of the institutional and/or national research committee(s) and with the Helsinki Declaration (as revised in 2013). Written informed consent was obtained from the patient families. 
Open Access Statement: This is an Open Access article distributed in accordance with the Creative Commons Attribution-NonCommercial-NoDerivs 4.0 International License (CC BY-NC-ND 4.0), which permits the noncommercial replication and distribution of the article with the strict proviso that no changes or edits are made and the original work is properly cited (including links to both the formal publication through the relevant DOI and the license). See: https://creativecommons.org/licenses/by-nc-nd/4.0/.

\section{References}

1. Poullis M, Poullis A. Biliptysis caused by a bronchobiliary fistula. J Thorac Cardiovasc Surg 1999;118:971-2.

2. Prodromos $\mathrm{P}$, Condilis N. Thoracobiliary fistula. A rare complication of thoracoabdominal trauma. Ann Ital Chir 2008;80:467-70

3. Gugenheim J, Ciardullo M, Traynor O, et al. Bronchobiliary fistulas in adults. Ann Surg

Cite this article as: Suh JH, Park CB. Bronchopleurobiliary fistula following right lower lobectomy in a patient with prior hepatic abscess: a case report. Ann Transl Med 2020;8(21):1464. doi: $10.21037 /$ atm-20-2776
1988;207:90-4.

4. Crnjac A, Pivec V, Ivanecz A. Thoracobiliary fistulas: literature review and a case report of fistula closure with omentum majus. Radiol Oncol 2013;47:77-85.

5. Liao GQ, Wang H, Zhu GY, et al. Management of acquired bronchobiliary fistula: A systemic literature review of 68 cases published in 30 years. World J Gastroenterol 2011;17:3842-9.

6. Ivatury RR, O'Shea J, Rohman M. Post-traumatic thoracobiliary fistula. J Trauma 1984;24:438-42.

7. Temes RT, Davis MS, Follis FM, et al. Videothoracoscopic treatment of hepatic hydrothorax. Ann thorac Surg 1997;64:1468-9.

8. Chua HK, Allen MS, Deschamps C, et al. Bronchobiliary fistula: Principles of management. Ann Thorac Surg 2000;70:1392-4.

9. Kontoravdis N, Panagiotopoulos N, Lawrence D. The challenging management of hepatopulmonary fistulas. J Thorac Dis 2014;6:1336-9. 